\title{
Diffraction des rayons $X$ en incidence rasante pour l'analyse des transformations structurales de surfaces d'aciers induites par implantation ionique multiple
}

\author{
P. Andreazza, X. de Buchere et R. Erre \\ Centre de Recherche sur la Matière Divisée, UMR 0131, Université d'Orléans, CNRS, BP. 6759, \\ 45067 Orléans cedex 02, France
}

\begin{abstract}
Résumé : La diffraction des rayons $\mathrm{X}$ en incidence rasante (GIXD) est une technique qui permet de déterminer la distribution en profondeur des differentes phases cristallines composant la surface d'un solide, et ceci en faisant varier la pénétration des rayons $X$ avec l'angle d'incidence. Les transformations structurales des surfaces d'aciers ont été étudiées par cette technique, dans le cas de l'implantation ionique multi-éléments, et ceci dans le but de comprendre les effets couples de l'implantation du silicium et de l'azote sur la structure de l'acier et sur son comportement électrochimique. De plus, un commentaire est présenté sur l'intêrêt et les limitations de la technique GIXD, notamment sur un dispositif de laboratoire.
\end{abstract}

Abstract : The Grazing Incidence X-ray Diffraction (GIXD) allows the determination of the in-depth distribution of various crystalline phases, which compose the surface of a solid, by varying the penetration depth with $X$-ray incidence angle. Surface transformations of stainless steel have been investigated with this technique, in the case of single- and multi-elements ionic implantation, in order to understand the coupled effects of silicium and nitrogen at microstructural level and in electrochemical behaviour. Interests and limits of laboratory GIXD technique are presented.

\section{INTRODUCTION}

L'intérêt grandissant pour l'analyse structurale de surface ou d'une certaine épaisseur proche de la surface d'un matériau a permis l'essor depuis quelques années de la diffraction des rayons X en incidence rasante (GIXD - Grazing Incidence X-Ray Diffraction) [1,2]. Plus particulièrement, cette technique non destructive a ouvert de nouvelles perspectives dans l'étude des modifications de l'organisation cristalline de surfaces d'aciers [3], dues au processus d'implantation ionique, notamment dans le but de comprendre l'influence de ces modifications sur les propriétés tribologiques des aciers.

La diffraction des rayons $\mathrm{X}$ en incidence rasante permet de déterminer la distribution en profondeur des différentes phases cristallines composant la surface d'un solide, et ceci en faisant varier la pénétration des rayons $\mathrm{X}$ avec l'angle d'incidence. Le développement de cette technique $[4,5]$ est en fait largement dû aux possibilités offertes par le rayonnement synchrotron (source intense de photons, longueur d'onde accordable, faible divergence du faisceau, ...), mais bien que soumise à de fortes limitations, l'utilisation en laboratoire est possible, particulièrement dans le cas d'échantillons polycristallins obtenus par traitement de surface ou par dépôt.

Dans cet article, nous presentons l'apport de la technique GIXD dans l'étude structurale des effets de l'implantation multiple d'ions (silicium et azote) sur des aciers, dans le but d'optimiser leurs propriétés électrochimiques. L'objectif global de l'étude consiste à améliorer la biocompatiblité et à accrôtre la durabilité des aciers employés dans le milieu biologique humain (prothèse destinée à être implantée dans le corps humain). 
Le comportement en corrosion localisée des aciers implantés dans un tel milieu a été notre principal critère de sélection des éléments d'implantation $(\mathrm{Ti}, \mathrm{Mo}, \mathrm{Si}, \mathrm{N}, \mathrm{C} \ldots)$ et de leurs associations, notament dans le cas du molybdène et du carbone [6]. Ainsi, nous ne présentons dans cet article que les implantations ( $\mathrm{Si}$ et $\mathrm{N}$ ) qui ont permis une amélioration notable du comportement électrochimique.

Enfin, cette étude GIXD a été menée de manière très complémentaire avec d'autres techniques d'analyse "destructives", la spectrométrie de photo-électrons (XPS) couplée à un dispositif d'abrasion ionique et la spectroscopie à décharge luminescente (GDOS) [7].

\section{METHODE DE DIFFRACTION EN INCIDENCE RASANTE}

\subsection{Principe}

L'intérêt d'une technique d'analyse de surface, se mesure à sa capacité à isoler les contributions superficielles des contributions de profondeur du matériau. Dans le cadre des rayons $\mathrm{X}$, nous pouvons faire varier la profondeur sondée entre quelques nanomètres et quelques micromètres en ajustant l'angle d'attaque $\alpha$ des rayons $\mathrm{X}$ sur la surface du solide à analyser.

Ainsi parce que l'indice de réfraction des solides dans la gamme des rayons $\mathrm{X}$ est faiblement inférieur à l'unité (écart de $10^{-5}-10^{-6}$ ), une réflexion spéculaire ou quasi-totale apparaît pour des angles d'incidence de quelques dixièmes de degré. A de tels angles, l'onde transmise est évanescente et sa profondeur de pénétration est très faible (quelques nanomètres, dépendant du matériau et de la longueur d'onde des rayons $X$ ), mais celle-çi augmente très rapidement dès que l'angle d'incidence s'éloigne de cette valeur critique $\alpha_{c}$ de réflexion totale [8] (Figure1).

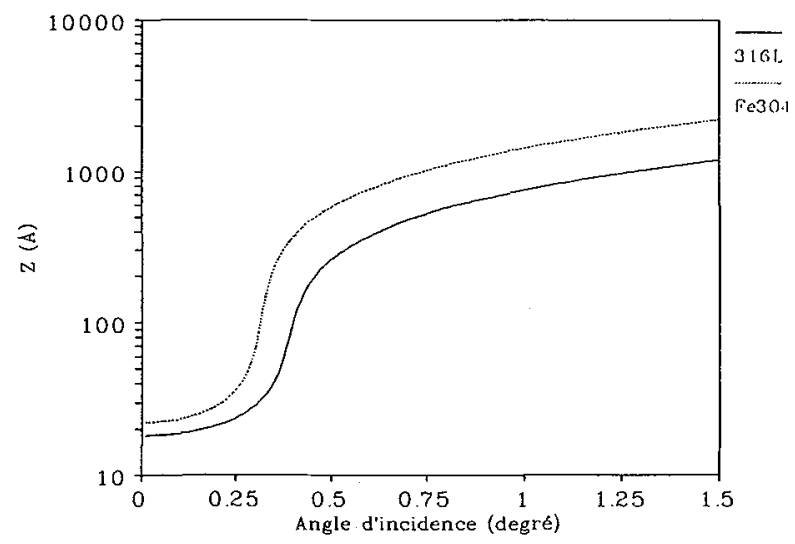

Figure 1: Evolution de la profondeur de pénétration des rayons $X\left(\lambda_{\mathrm{CuK} \alpha}=1,54178 \AA\right)$ en fonction de l'angle d'incidence dans le cas de l'acier 316 et de l'oxyde $\mathrm{Fe}_{3} \mathrm{O}_{4}$.

RX penetration depth evolution versus incidence angle for $316 \mathrm{~L}$ stainless steel and $\mathrm{Fe}_{3} \mathrm{O}_{4}$ oxide.

A partir d'une telle propriété, l'analyse de surface d'un solide plan est alors possible par une détection de la diffraction ou de la fluorescence de la surface sous incidence rasante. Dans le cas de la diffraction, une telle technique permet dans son principe à la fois l'étude structurale 
bidimensionnelle de couches très superficielles (monocouches), par exemple épitaxiées sur des monocristaux [4] et l'analyse tridimensionnelle d'échantillons polycristallins tels que les aciers implantés [3]. Dans les deux cas, l'incidence est rasante, mais la détection de l'onde diffractée se fait respectivement, dans le plan de l'échantillon en émergence rasante, ou, dans le plan perpendiculaire en émergence oblique. Nous disposons ainsi, d'une sonde analysant l'organisation cristalline en profondeur, de la surface jusqu'aux couches internes du matériau.

\subsection{Dispositifs expérimentaux et paramètres de mesure}

Le système instrumental est un diffractomètre horizontal $(G)$ deux cercles PHILIPS couplé avec un porte-échantillon goniométrique deux berceaux - trois translations. La source de rayons $\mathrm{X}$ est un tube scelle à foyer fin lineaire (TRX) émettant après filtrage (F) la raie CuK $\alpha(\lambda=$ $1,54178 \AA$ ). La géométrie du dispositif (figure 2) est basée sur la méthode du faisceau quasiparallèle. L'optique primaire (fente de Soller horizontales (FS1) et fente de divergence (F1)) permet de limiter la divergence horizontale $\left(\Leftrightarrow 0,2^{\circ}\right)$ mais surtout la divergence verticale $\left(<0,03^{\circ}\right)$ du faisceau, qui conditionne la sélectivité en profondeur de l'analyse GIXD. L'optique secondaire de réception (fentes de Soller à divergence verticale (FS2), monochromateur graphite plan (MGP) et détecteur (D)) assure la sélection et la détection du faisceau diffracté.

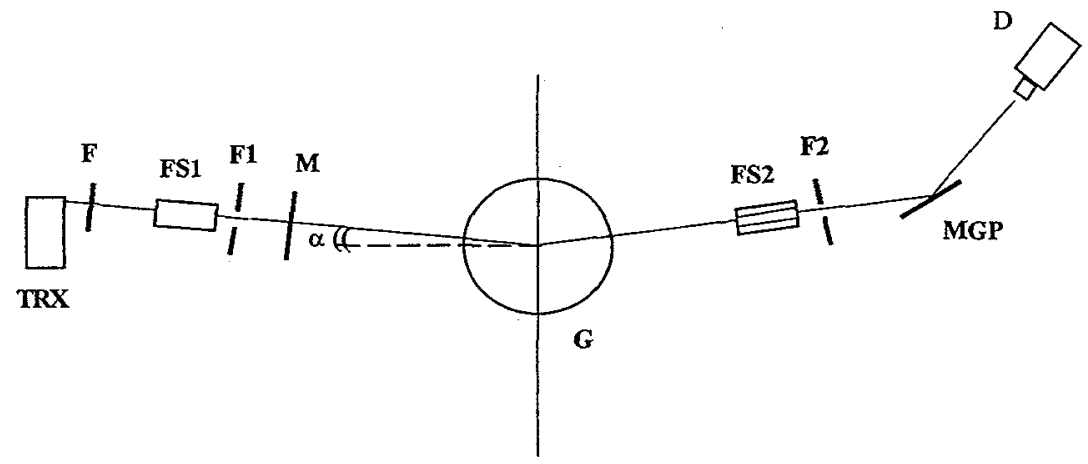

Figure 2: Dispositif expérimental de diffraction GLXD. GLXD experimental set-up.

A partir de ce dispositif, l'analyse des surfaces d'aciers implantés a été effectuée à différents angles d'incidence de $0,2^{\circ}$ à $1,5^{\circ}$ de manière à mettre en évidence l'évolution de la microstructure en fonction de la profondeur. Cette profondeur de pénétration étant très liée à la nature et à la densité du matériau, son estimation devient très imprécise en incidence très rasante ( $\alpha<0,5^{\circ}$ proche de l'angle critique), notamment lors de la présence de couches superficielles très oxydées (Figure 1). Enfin dans l'interprétation des diffractogrammes GLXD, nous avons tenu compte de l'effet de réfraction quantifié expérimentalement grâce à un échantillon de référence non-implanté ayant le même état de surface et de planéitté. En effet pour des angles d'incidence aussi faibles, l'écart d'indice (1-n) n'est plus négligeable et l'onde transmise est réfractée introduisant une erreur dans l'évaluation de l'angle de diffraction $2 \theta$. 
Un dispositif annexe de diffraction classique nous a permis également d'effectuer des mesures en configuration symétrique $(\theta-2 \theta)$ convergente, notamment pour caractériser la matrice austénitique des échantillons et mesurer la sensibilité du système GIXD.

\section{ANALYSE STRUCTURALE DE SURFACES D'ACIERS IMPLANTÉS}

\subsection{Caractéristiques des échantillons et paramètres d'implantation}

L'acier austénitique utilisé pour cette étude est de type 316L (Norme biomédicale) avec la composition suivante: $\mathrm{Cr}: 18 \%$, Ni:12\%, Mo:2,5\%, C<0,02\% et le reste en Fe. La présence de molybdène et le faible pourcentage de carbone sont destinés à augmenter la résistance à la corrosion. Les échantillons ont été préparés sous forme de pastilles de $11 \mathrm{~mm}$ de diamètre polis mécaniquement jusqu'à l'obtention des rugosités moyennes de $30 \AA$ et "peak to valley" de $130 \AA$ estimées par AFM (microscopie à force atomique).

Les implantations ioniques ont été effectuées au Département de Physique des Matériaux de Villeurbanne sur un accélếrateur à implantation de tension maximale de $200 \mathrm{kV}$, à température ambiante. Le vide dans la chambre d'implantation était de l'ordre de $10^{-6} \mathrm{mbar}$.

Dans les trois types d'échantillons implantés (Tableau I), la dose et l'énergie d'implantation du silicium sont identiques $\left(10^{17} \mathrm{at} / \mathrm{cm}^{2}, 140 \mathrm{keV}\right)$ avec une double implantation pour la série SIN2 $\left(5.10^{16} \mathrm{at} / \mathrm{cm}^{2}, 70 \mathrm{keV}\right)$. En ce qui concerne l'implantation de l'azote, les conditions sont différentes (Tableau I). Mais dans tous les cas, ces paramètres ont été choisis conformément à la théorie Lindhard, Scharff et Schiott (LSS) pour que les concentrations en silicium et azote en profondeur présentent une distribution pseudo-gaussienne centrée sur le même maximum noté $R_{P}$ de $900 \AA$ environ avec une seconde distribution centrée sur $500 \AA$ environ pour la série SIN2.

Tableau 1: Paramètres d'implantation du silicium et de l'azote dans les trois types d'echantillons analyses. Silicon and nitrogen implantation parameters for the three types of samples.

\begin{tabular}{|cccccc|}
\hline & \multicolumn{2}{c}{ Silicium } & \multicolumn{2}{c|}{ Azote } \\
Dose & $\begin{array}{c}\text { Energie } \\
\mathrm{keV}\end{array}$ & $\begin{array}{c}\text { Dose } \\
\mathrm{at} / \mathrm{cm}^{2}\end{array}$ & $\begin{array}{c}\text { Energie } \\
\mathrm{keV}\end{array}$ & $\begin{array}{c}\mathrm{R}_{\mathrm{P}} \\
\AA\end{array}$ \\
SI1 & $10^{17}$ & 140 & & & \\
SIN1 & $10^{17}$ & 140 & $1,3310^{17}$ & 80 & $\approx 900$ \\
SIN2 & $10^{17}$ & 140 & $210^{17}$ & 80 & $\approx 900$ \\
& $+510^{16}$ & 70 & $+10^{17}$ & 40 & $\approx 500$ \\
& & & & & \\
\hline
\end{tabular}

\subsection{Résultats des analyses GIXD}

\subsubsection{Acier de référence et corrections.}

Les différents spectres relatifs à ces échantillons ne mettent en évidence que la présence d'austénite $\gamma(\mathrm{Fm} 3 \mathrm{~m})$, quels que soient le dispositif de diffraction ( $\theta-2 \theta$ ou rasant), ou l'angle 
d'incidence utilisé. Aucun oxyde n'est détecté en extrême surface $\left(\alpha=0,2^{\circ}\right)$ bien que l'analyse XPS en surface fasse apparaitre une couche d'oxydes de $20 \AA$ très riche en chrome.

Cependant, un effet d'orientation préférentielle des plans (220) parallèlement à la surface est nettement révélé pour tous les échantillons, certainement du à la mise en forme de l'acier. Le paramètre de maille de cette phase $\gamma(\mathrm{a}=3,598 \AA)$ est légèrement supérieur à celle de la fiche JCPDS ( $a=3,591 \AA$ pour l'acier $304 \mathrm{~L}$ ). En effet l'encombrement du à la présence de $2 \%$ de molybdène dans la nuance $316 \mathrm{~L}$ correspond effectivement à cette dilatation de paramètre. Les principales réflexions de la phase austénitique $\gamma(316 \mathrm{~L})$ dans la gamme angulaire que nous avons étudiée sont $2 \theta_{111}=43,58^{\circ}, 2 \theta_{220}=50,79^{\circ}$ et $2 \theta_{200}=74,70^{\circ}$. Une évaluation de la taille des domaines de cohérence, couplée avec une observation par microscopie électronique donne sur tous les échantillons étudiés des valeurs supérieures à $0,5 \mu \mathrm{m}$.

Enfin, des mesures en diffraction en incidence rasante ont permis de déterminer les effets de refraction sur les trois raies principales pour les incidences $\alpha=0,2^{\circ}, 0,5^{\circ}$ et $1,5^{\circ}$, 1'angle critique pour un acier $316 \mathrm{~L}$ étant $\alpha_{c}=0,38^{\circ}$.

\subsubsection{Surface d'acier implanté Si (SII)}

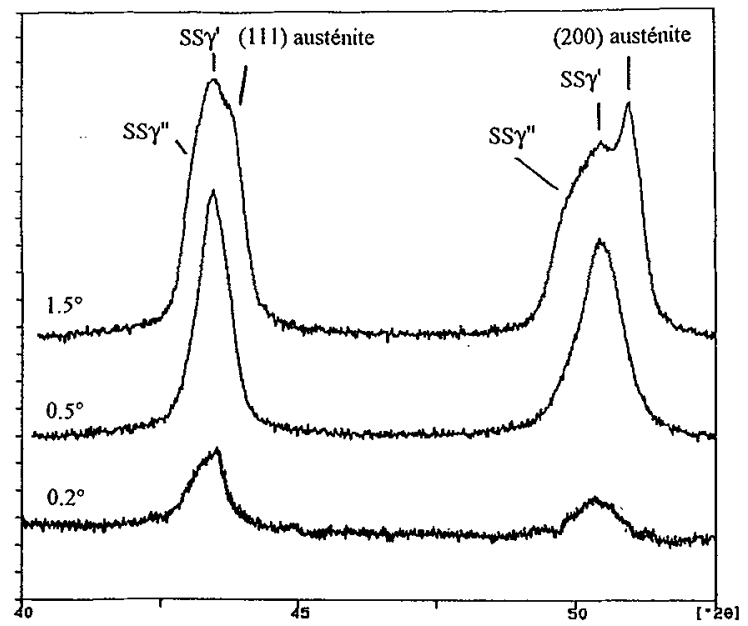

Figure 3: Spectres GIXD de l'échantillon SI1 (implanté Si) pour différents angles d'incidence $\left(0,2^{\circ}, 0,5^{\circ}\right.$ et $\left.1,5^{\circ}\right)$ correspondant respectivement aux profondeurs d'analyse $(<50 \AA, \approx 250 \AA$ et $\approx 1200 \AA)$. Les spectres sont en echelle carrete et translatés pour plus de clarte. ( $S S=$ Solution Solide)

GIXD spectra of SI1 sample (implanted Si) at different angles $\left(0,2^{\circ}, 0,5^{\circ}, 1,5^{\circ}\right)$ corresponding respectively to $<50 \AA, \approx 250 \AA$ and $\approx 1200 \AA$ analysis depth. (SS= Solid Solution)

Cet échantillon mono-implanté a été analysé dans le but d'observer les transformations structurales induites par le silicium seul. Les différents spectres de diffraction GIXD (figure 3) montrent la présence d'une solution solide (SS) austénitique de silicium. En effet, la forme des trois principales raies de l'austénite est très différente de celle de la matrice non-implantée et évolue en fonction de la profondeur d'analyse. Compte-tenu de l'effet de réfraction, les raies obtenues pour l'incidence très rasante $\left(\alpha=0,2^{\circ}\right)$ présentent un décalage vers les faibles angles 
résultant de l'insertion de silicium dans la maille austénitique. Cette interprétation est confirmée

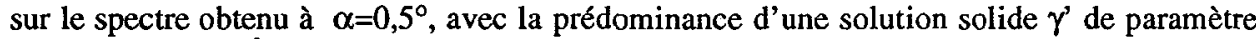
cristallin $a=3,625 \AA$ et la présence d'un épaulement très minoritaire $\gamma^{\prime \prime}$ aux faibles angles sur les trois raies, qui pourrait être du à des taux d'insertion de silicium plus élevés. Cette "seconde" solution solide devient quantitativement aussi abondante que la précédente pour $\alpha=1,5^{\circ}$.

Nous observons de plus l'apparition de la matrice austénitique correspondant au paramètre de l'échantillon de référence, donc n'ayant subi aucune altération décelable de type insertion. Cette observation nous confirme que l'information structurale recueillie correspond pour cette angle d'incidence $\left(\alpha=1,5^{\circ}\right)$ à une profondeur d'analyse supérieure à l'épaisseur modifiée par l'implantation. Aucune autre phase n'a été détectée.

\subsubsection{Surface d'acier implanté Si $+N(S I N I)$}

Cette double implantation d'atomes de silicium et d'azote a induit de fortes modifications dans la surface de l'acier. La transformation la plus remarquable est la présence quasi-exclusive (figure 4) d'oxyde de fer $\mathrm{Fe}_{3} \mathrm{O}_{4}$ en extrême surface $\left(\alpha=0,2^{\circ}\right)$. Les cinq raies les plus intenses qui le caractérisent, sont observées sans effet de texture notable, alors que les raies de plus faibles intensités n'apparaissent qu'à partir de $\alpha=0,5^{\circ}$.

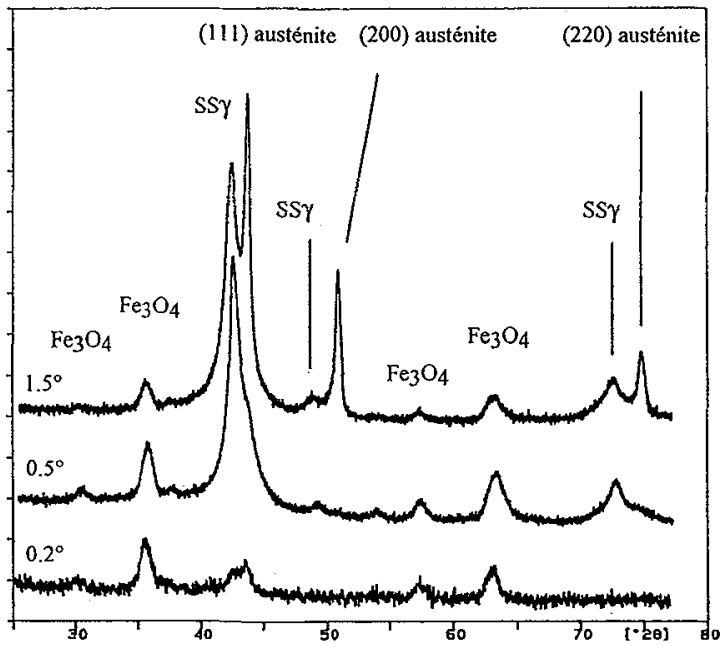

Figure 4: Spectres de diffraction GIXD de l'échantillon SIN1 (implanté $\mathrm{Si}+\mathrm{N}$ ) pour différents angles d'incidence $\left(0,2^{\circ}, 0,5^{\circ}\right.$ et $\left.1,5^{\circ}\right)$ correspondant respectivement aux profondeurs d'analyse $(<50 \AA, \approx 500 \AA$ et $\approx 1500 \AA)$. Les spectres sont en échelle carrée et translatés pour plus de clarté.

GIXD spectra of SIN1 sample (implanted $\mathrm{Si}+\mathrm{N})$ at different angles $\left(0,2^{\circ}, 0,5^{\circ}, 1,5^{\circ}\right)$ corresponding respectively to $<50 \AA, \approx 500 \AA$ and $\approx 1500 \AA$ analysis depth.

Mise à part la présence de cet oxyde, seuls les domaines angulaires proches des trois raies principales de l'austénite montrent une évolution en fonction de la profondeur. En effet, trois raies décalées vers les faibles angles par rapport à l'austénite $\gamma$ semblent être significatives de la 
présence d'une solution solide d'insertion silicium et/ou azote, dont le paramètre est centré sur $a=3,713 \AA$. Malgré cela, l'importance du domaine de diffraction entre $40^{\circ}$ et $45^{\circ}$ (figure 4) en intensité et en largeur ne peut pas être totalement attribuée à cette solution solide. Aucune autre réflexion aussi significative n'a pu être mise en évidence. La raie principale à $2 \theta=42,3^{\circ}$ comportant une base très large pourrait contenir les contributions des réflexions principales de nitrure de chrome (CrN) ou de fer, ou des raies (101) et dans une moindre mesure (110) de la martensite tétragonale, notamment si l'on considère également la structure assez complexe située autour de $2 \theta=63^{\circ}$. Dans tous les cas de figure, seule une orientation préférentielle associée à une combinaison de contributions peut expliquer une prépondérance si nette de ce domaine de diffraction, particulièrement sur le spectre à $\alpha=0,5^{\circ}$ (figure 4). Cette difficulté d'interprétation pourrait être due aux limitations de notre dispositif en terme de résolution, donc de séparation des contributions trop proches angulairement.

En outre, on observe l'apparition à $\alpha=1,5^{\circ}$ de la matrice austénitique très faiblement révélée à $\alpha=0,5^{\circ}$. Notons que compte-tenu de la présence d'une couche d'oxyde de surface sur une épaisseur importante (200-300 $\AA$ ), l'estimation initiale des profondeurs de pénétration est décalée pour $\alpha=0,5^{\circ}$ et $1,5^{\circ}$ (respectivement $\approx 500 \AA$ et $1500 \AA$ ).

\section{2. furface d'acier implanté $(S i+N)+(S i+N)(S I N 2)$}

L'originalité de cet échantillon est de ne montrer aucune raie nettement résolue, mis à part les réflexions principales de la matrice austénitique à $\alpha=1,5^{\circ}$ (figure 5). En revanche, les domaines angulaires de $38^{\circ}$ à $52^{\circ}$, et de $68^{\circ}$ à $100^{\circ}$ dans une moindre mesure, présentent une structure très complexe déjà visible à $\alpha=0,5^{\circ}$, constituée de larges raies (décalées par rapport aux raies de l'austénite $\gamma$ ) associées à de nombreux épaulements.

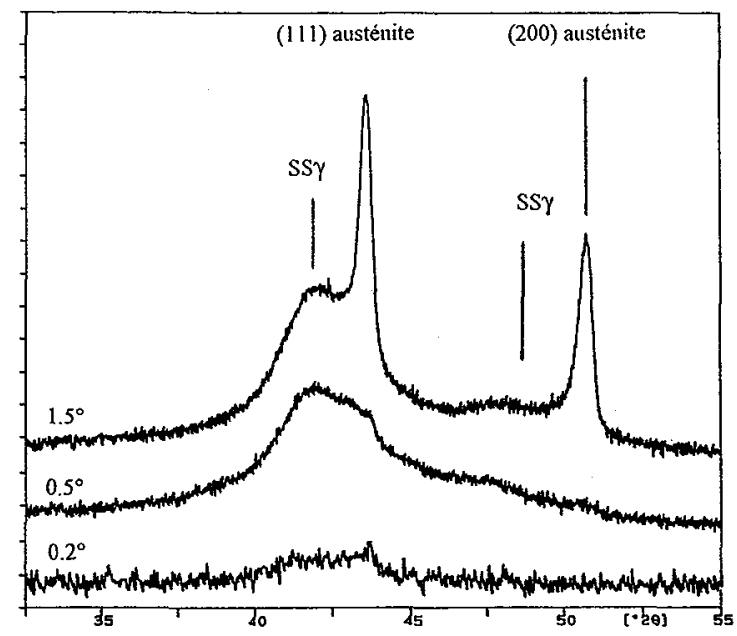

Figure 5: Spectres de diffraction GIXD de l'echantillon SIN2 (implanté $\mathrm{Si}+\mathrm{N}+\mathrm{Si}+\mathrm{N}$ ) pour différents angles $d^{\prime}$ 'incidence $\left(0,2^{\circ}, 0,5^{\circ}\right.$ et $\left.1,5^{\circ}\right)$ correspondant respectivement aux profondeurs d'analyse $(<50 \AA, \approx 250 \AA$ et $\approx 1200 \AA$ ). Les spectres sont en échelle carree et translatés pour plus de clarté.

GIXD spectra of SIN2 sample (implanted $\mathrm{Si}+\mathrm{N}+\mathrm{Si}+\mathrm{N})$ at different angles $\left(0,2^{\circ}, 0,5^{\circ}, 1,5^{\circ}\right)$ corresponding respectively to $<50 \AA, \approx 250 \AA$ and $\approx 1200 \AA$ analysis depth. 
Malheureusement, bien que la présence systématique de contributions d'une ou plusieurs solutions solides d'insertion azote-silicium soit certaine, une interprétation sur la nature des autres contributions serait très hasardeuses

Néanmoins, la largeur des contributions est significative d'une diminution drastique de la taille des domaines de coherence par rapport à la matrice, mais aussi par rapport aux implantations SI1 et SIN1. Cette couche de surface ne ressemble plus à un acier.

\subsection{Discussion}

A partir des résultats précédents, il apparaît que les transformations structurales induites par l'implantation de silicium seul (SI1) se limitent à l'apparition de solutions solides austénitiques d'insertion, pour lesquelles le taux d'insertion de $\mathrm{Si}$ augmenterait avec la profondeur jusqu'à la valeur $R_{p}$. Cette interprétation est conforme aux profils de concentration obtenus en XPS, qui montrent une concentration constante $(\approx 5 \%)$ en silicium sur une épaisseur de $400 \AA$, puis une large distribution centrée sur $900 \AA$. Ainsi, on observe en GIXD que le taux d'insertion dominant (phase $\gamma^{\prime}$ ) correspond aux premières centaines d'angström. Plus en profondeur, d'autres contributions apparaissent correspondant à la fois, à la zone de concentration maximale en $\mathrm{Si}(900 \AA)$ donc de fort taux d'insertion et à une zone de faible concentration proche de la matrice non-implantée.

Dans le cas de l'échantillon implanté $\mathrm{Si}+\mathrm{N}$ (SIN1), les profils XPS et l'analyse GDOS font apparaître une couche oxydée très épaisse $(\approx 250 \AA)$ mise en évidence en GLXD, certainement due à des effets conjugués de détérioration de la qualité du vide et d'effets de température induits par l'implantation.

En comparaison par rapport à l'échantillon SI1 (figure 6), la position angulaire de la solution solide (observable également sur les 2 autres raies (200) et (220)) montrent l'effet supplémentaire de l'insertion d'azote. En utilisant les résultats de Rauschenbach et Kolitsh [9] sur l'évolution du paramètre de maille de l'austénite en fonction du taux d'insertion d'azote, on obtient la relation linéaire:

$$
a=\left(a_{0}+0,008 \% \mathrm{~N}\right) \AA
$$

où an est le paramètre de maille de l'acier avant l'implantation d'azote.

$\mathrm{Si}$, à partir du paramètre de maille de la solution solide $(a=3,71 \AA)$ évalué d'après les positions angulaires des trois raies, nous calculons le pourcentage sans tenir compte de l'insertion du silicium, le taux d'insertion d'azote obtenu est de $14 \%$, ce qui serait supérieur à la concentration maximale $(\approx 10 \%)$ détectée par XPS. Mais le silicium étant implanté avant l'azote, nous pouvons admettre qu'il se soit comporté comme dans l'échantillon SIl (même paramètres d'implantation) créant une solution solide $\gamma^{\prime}(a=3,64 \AA)$ dans un premier temps, qui subissant ensuite l'effet d'insertion de l'azote lors de son implantation, donne la solution solide mixte mise en évidence. A partir de la valeur moyenne du paramètre de la solution $\boldsymbol{\gamma}^{\prime}$, nous pouvons estimer un pourcentage d'insertion d'azote de $9 \%$.

Notons également que d'autres auteurs, sur différents aciers $[10,11]$, ont constaté pour une implantation d'azote seul dans des conditions similaires, l'apparition de solutions solides à taux d'insertion élevés (>15\%), mais également de nitrure de chrome dont la présence n'est pas sure dans notre cas.

Enfin, comparativement (figure 6), l'implantation multiple SIN2 introduit un bouleversement structural important dans la zone de surface. Les analyses XPS et GDOS montrent une répartition gaussienne très large $(\approx 1500 \AA)$ du silicium et de l'azote centrée sur $900 \AA$ et une redistribution des éléments constitutifs de l'acier totalement différente des autres 
échantillons. Le fer est prépondérant $(80 \%)$ en surface $(<400 \AA)$ au détriment de tout les autres éléments, notamment du chrome, alors que dans la gaussienne d'implantation, la concentration de chrome augmente (20\%) au détriment du fer $(30 \%)$, avant de retrouver les proportions de l'acier. On observe une véritable démixtion de l'acier. Les causes de cette ségrégation pourraient provenir de l'effet conjugué de la température comme accélérateur de diffusion et de la création d'un gradient de lacunes induit par effet balistique lors de la multi-implantation.

En résumé, la présence de solutions solides d'insertion a été mise en évidence pour tous les échantillons, sans qu'aucune autre phase, excepté l'oxyde de surface, n'ait étê révélé.

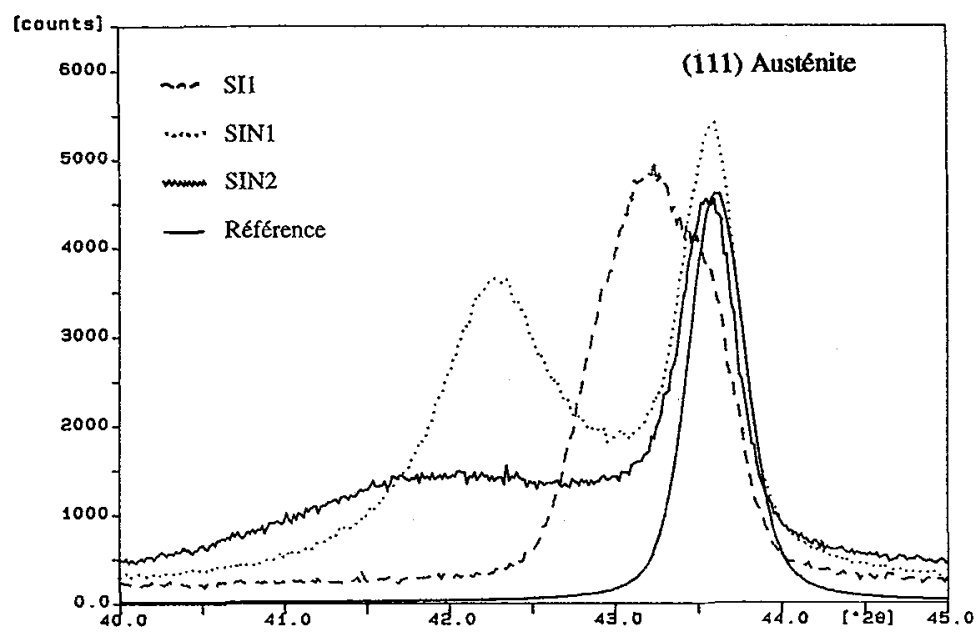

Figure 6: Spectres GIXD des échantillons de reférence, SI1, SIN1 et SIN2 pour l'angle d'incidence $\alpha=1,5^{\circ}$. Les spectres sont en échelle linéaire (l'intensité du spectre de référence a êté divisée par 10). GIXD spectra of reference, SI1, SIN1 and SIN2 samples at $\alpha=1,5^{\circ}$.

\section{CONCLUSION}

Les résultats précédents mettent en relief à la fois les avantages de la méthode de diffraction des rayons $X$ en incidence rasante et ses limitations, ou plus précisément les limitations du dispositif GIXD que nous avons utilisé.

En effet, cette méthode a permis de révéler la présence de phases polycristallines (stables ou métastables) de "surface" sur des profondeurs de quelques dizaines ou centaines d'angström, invisible avec un dispositif classique de diffraction $\theta-2 \theta$. Elle a également permis de montrer l'évolution en fonction de la profondeur des phases qui composent les surfaces d'aciers implantés.

En revanche, nous avons pu apprécier la difficulté à séparer les contributions trop proches les unes des autres, notamment si les domaines de cohérence des phases détectées sont de très faibles dimensions. D'autre part, cette méthode est certainement peu adaptée aux échantillons très texturés du moins en configuration "incidence rasante - émergence non-rasante". De plus, l'intensité des rayons $\mathrm{X}$ disponible avec un dispositif à tube scellé est difficilement compatible avec des durées d'acquisition raisonnable, qui conditionnent l'importance du bruit statistique et la possibilité de balayer plus précisément le domaine angulaire d'incidence (c.a.d. la profondeur d'analyse). 
En résumé, malgré certaines limitations dans le domaine de la résolution et de l'intensité $\mathrm{X}$ disponible, cette méthode est effectivement bien adaptée à l'étude de l'organisation structurale des zones superficielles des solides polycristallins, et notamment des surfaces d'aciers modifiees par implantation ionique.

Enfin; l'accès à des informations quantitatives sur la distribution en profondeur des différentes phases serait certainement un complément appréciable à ce type d'étude, et particulièrement au regard des informations obtenues par XPS en érosion ionique.

\section{Références}

[1] Marra W.C., Eisenberger P., Cho A.Y., J. Appl. Phys. 50 (1979) 6927.

[2] Brunel M., De Bergevin, Act. Cryst. A42 (1986) 299.

[3] Arnaud Y., Brunel M., De Becdelievre A.M., Romand M., Thevenard P., Robelet M., Appl. Surf. Sc. 26 (1986) 12.

[4] Bohr J, Feidenhans'l R., Nielsen M., Toney M., Phys. Rev. Lett. 54 (1985) 1275.

[5] Pons F., Megtert S., Pivin J.C., J. Appl. Cryst. 21 (1988) 197.

[6] De Buchere X., Andreazza P., Andreazza-Vignolle C., Clinard C., Erre R., Surf. Coatings Tech. (1995) sous presse.

[7] De Buchere X., These de l'Université d'Orléans (1995)

[8] James R.W., The optical principles of the diffraction of X-rays, Cornell Univ. Press, Ithaca (1965).

[9] Rauschenbach B., Kolitsch A., Phys. Stat. Sol. (a) 80 (1983), 211-222.

[10] Cordier-Robert C., Bourdeau L., Magnin T.,Foct J., Mater. Letters 20 (1994) 113-118.

[11] Arnaud Y., Brunel M., Appl. Surf. Sc. 35 (1989) 345. 